\title{
Correction to: Current situation of cancer among adolescents and young adults in Japan
}

\author{
Akira Ohara $^{1}$ (1) $\cdot$ Tatsuro Furui $^{2} \cdot$ Chikako Shimizu $^{3} \cdot$ Seiichiro Ozono ${ }^{4} \cdot$ Kazuhito Yamamoto $^{5} \cdot$ Akira Kawai $^{6}$. \\ Ryohei Tatara $^{7} \cdot$ Akiko Higuchi $^{8} \cdot$ Keizo Horibe $^{9}$
}

Published online: 15 October 2018

(c) Japan Society of Clinical Oncology 2018

\section{Correction to: International Journal of Clinical Oncology https://doi.org/10.1007/s10147-018-1323-2}

In the original publication, Tables 4 and 5 have not been published in a readable format. The corrected clear version is given in this Correction.

The other errors and their corrections are given below. The publisher sincerely apologizes for the errors.

The original article has been corrected.

The original article can be found online at https://doi.org/10.1007/ s10147-018-1323-2.

Akira Ohara

aohara@med.toho-u.ac.jp

Tatsuro Furui

furui@gifu-u.ac.jp

Chikako Shimizu

cshimizu@ncc.go.jp

Seiichiro Ozono

oznsei@outlook.jp

Kazuhito Yamamoto

kyamamoto@aichi-cc.jp

Akira Kawai

akawai@ncc.go.jp

Ryohei Tatara

r-tatara@med.osakacity-hp.or.jp

Akiko Higuchi

hguchi@ccaj-found.or.jp

Keizo Horibe

horibek@nnh.hosp.go.jp

1 Department of Pediatrics, Toho University, 6-11-1, Omori-Nishi, Ota-ku, Tokyo 143-8541, Japan
2 Department of Obstetrics and Gynecology, Gifu University Graduate School of Medicine, 1-6-1 Yanagido, Gifu, Gifu 501-1194, Japan

3 Department of Breast and Medical University, National Cancer Center Hospital, 5-1-1, Tsukiji, Chuo-ku, Tokyo 104-0045, Japan

4 Department of Urology, Hamamatsu University School of Medicine, 1-20-1 Handayama Higashi-ku, Hamamatsu, Shizuoka 431-3192, Japan

5 Department of Hematology and Cell Therapy, Aichi Cancer Center, 1-1 Kanokoden, Chikusa-ku, Nagoya, Aichi 464-8681, Japan

6 Department of Musculoskeletal Oncology, National Cancer Center Hospital, 5-1-1, Tsukiji, Chuo-ku, Tokyo 104-0045, Japan

7 Department of Palliative Medicine, Osaka City Medical Hospital, 2-13-22, Miyakojima-hondori, Miyakojima-ku, Osaka 534-0021, Japan

8 Children's Cancer Association of Japan, 1-3-12, Asakusabashi, Taito-ku, Tokyo 111-0053, Japan

9 Clinical Research Center, National Hospital Organization, Nagoya Medical Center, 4-1-1 Sannomaru, Naka-ku, Nagoya 460-0001, Japan 


\begin{tabular}{lllll}
\hline Page & Column & Line & In the original article & Should be \\
\hline 1 & 1 & 21 & approach (217 words) & approach \\
2 & 2 & 114 & 1481 & 1,481 \\
& 2 & 127 & 7587 & 7,587 \\
& Table 1 & & 1481 & 1,481 \\
& Table 1 & & 2209 & 2,209 \\
& Table 1 & & 4349 & 4,349 \\
& Table 1 & 6674 & 6,674 \\
3 & Table 2 & & 4992 & 4,992 \\
& Table 2 & & 7587 & 7,587 \\
4 & Table 3 & 1077 & 1,077 \\
& Table 3 & & 8111 & 8,111 \\
& Table 3 & & 1734 & 1,734 \\
4 & Table 3 & 1726 & 1,726 \\
& Table 3 & & 1045 & 1,045 \\
5 & Table 3 & 1664 & 1,664 \\
5 & Table 3 & & 8111 & 8,111 \\
5 & Table 3 & 2578 & 2,578 \\
5 & Table 3 & 5500 & 5,500 \\
5 & Table 3 & 4334 & 4,334 \\
5 & Table 3 & 3768 & 3,768 \\
5 & Table 3 & 4178 & 4,178 \\
8 & Table 4 & & th & the \\
9 & Table 5 & 1080 & 1,080 \\
\hline
\end{tabular}




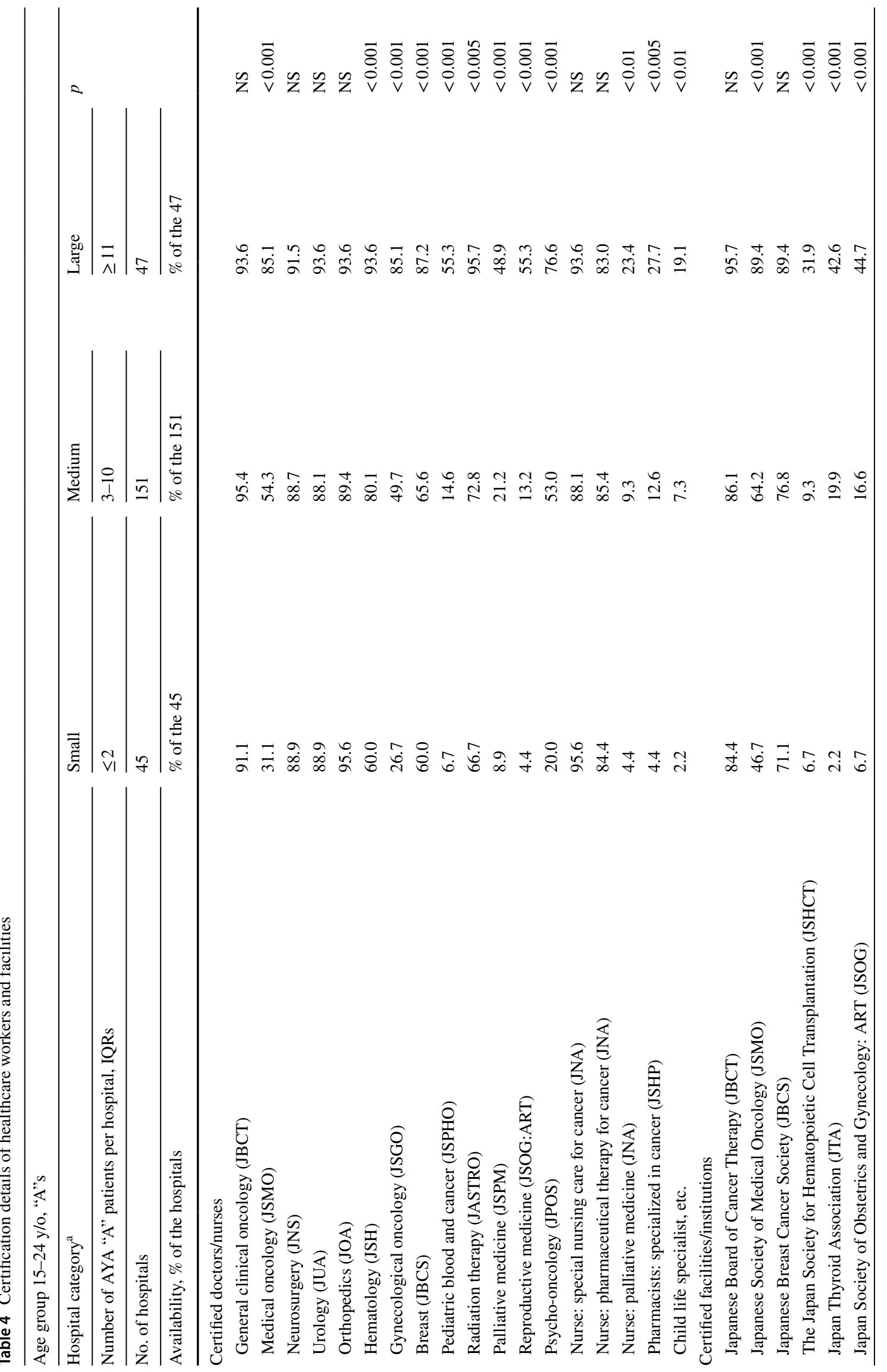




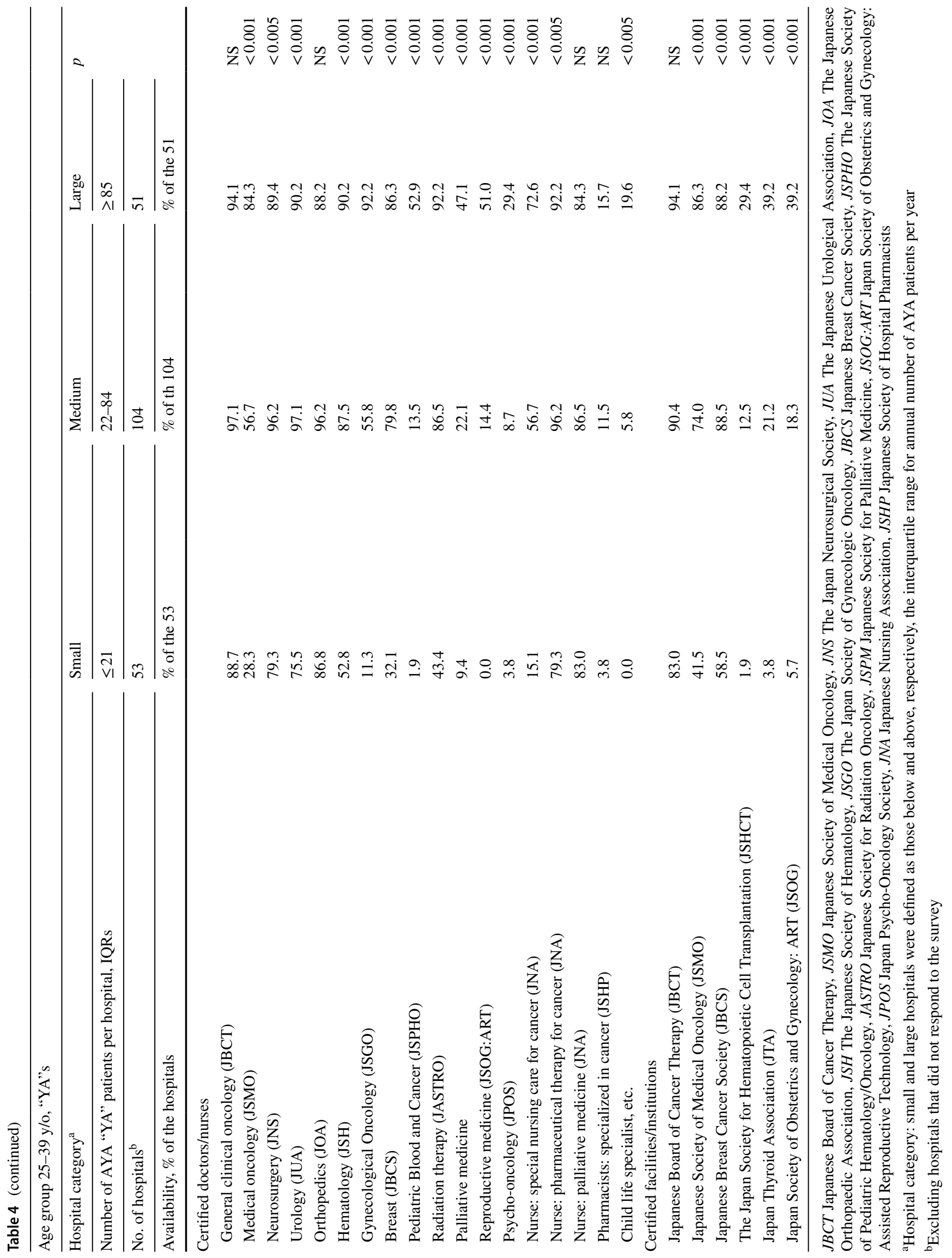


Table 5 Treatment centralization by cancer topography

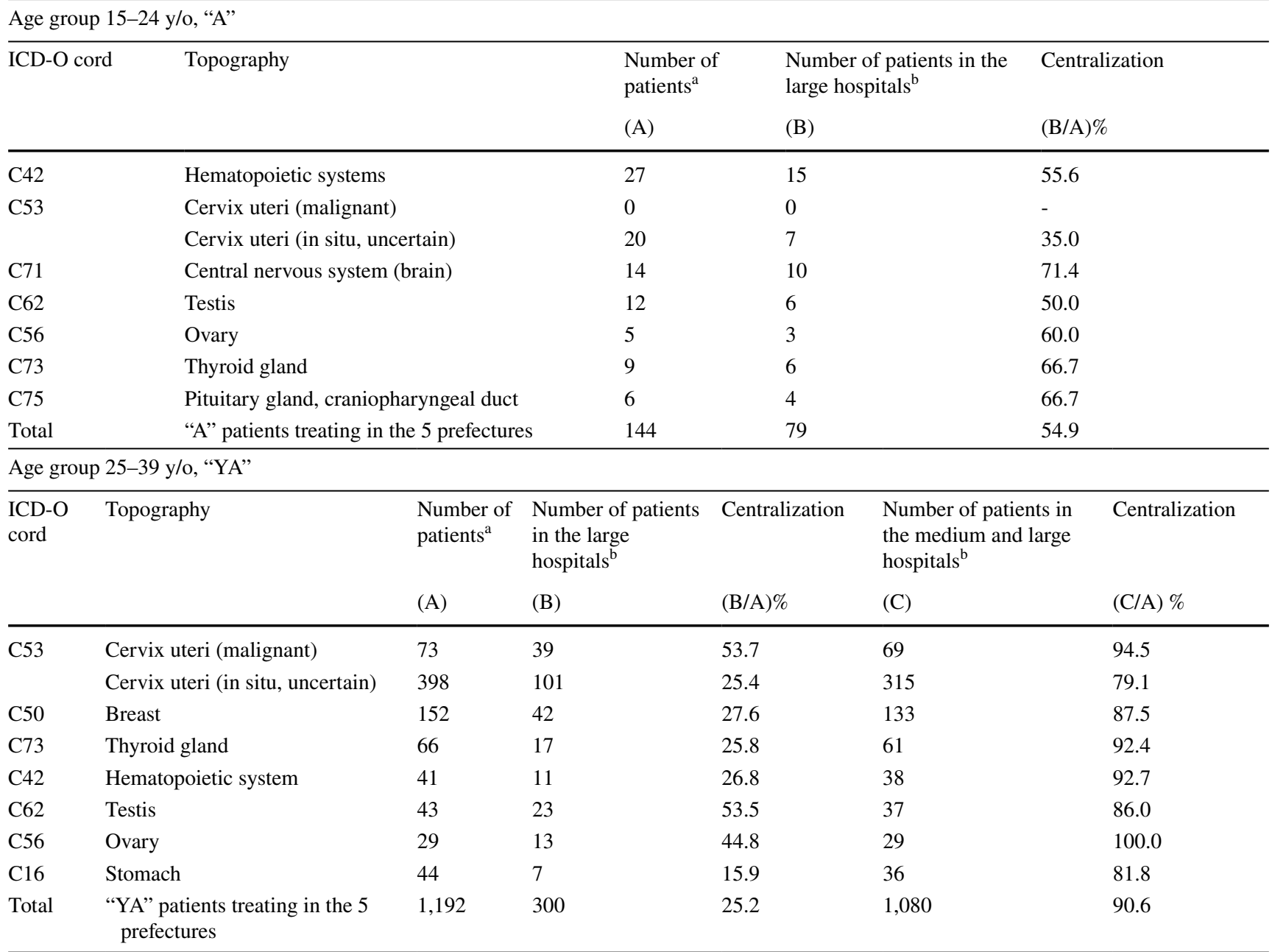

${ }^{a}$ Total number of patients treating in the 5 prefectures; Nagasaki, Okayama, Miyagi, Shimane, and Yamanashi

${ }^{\mathrm{b}}$ Hospitals in the 5 prefectures 